\title{
SMALL-POLARON MOBILITY IN NONSTOICHIOMETRIC CERIUM DIOXIDE
}

\author{
I. K. NAIK $†$ and T. Y. TIEN \\ Department of Materials and Metallurgical Engineering, The University of Michigan, Ann Arbor, MI 48109, U.S.A.
}

(Received 27 May 1977; accepted in revised form 22 September 1977)

\begin{abstract}
The high temperature drift mobility $\left(\mu_{d}\right)$ of charge carriers in nonstoichiometric cerium dioxide $\left(\mathrm{CeO}_{2_{-x}}\right)$ has been calculated by combining the electrical conductivity and nonstoichiometry data on the basis of the oxygen vacancy model with correct ionization state. The electrical conductivity was measured by a four-probe d.c. technique and the nonstoichiometry by thermogravimetric analysis. The dilute solution model of the point defects is valid up to $x=0.03$. From the magnitude of $\mu_{d}$ and its temperature dependence, the charge carriers in $\mathrm{CeO}_{2-x}$ are proposed to be small-polarons formed by localization of electrons at cerium sites and the charge transport process is proposed to occur by a hopping mechanism. The observed temperature dependence of $\mu_{d}$ is in accord with that derived by Holstcin and Friedman for small-polaron transport by the hopping mechanism. The activation energy of mobility is found to increase with increasing $x$ as expected for the hopping model.
\end{abstract}

\section{INTRODUCTION}

Electronic conduction in solid oxides can occur by motion of the charge carriers in a band or by a diffusionlike motion called hopping mechanism. The hopping mechanism is considered to apply to the low mobility oxide semiconductors at elevated temperatures and the charge carrier in this case is termed a small-polaron. It is the purpose of this paper to show experimental evidence for small-polarons and to demonstrate the hopping-type small-polaron mobility at elevated temperatures in nonstoichiometric cerium dioxide $\left(\mathrm{CeO}_{2-x}\right)$.

From the electrical conductivity and thermodynamic measurements at temperatures above $700^{\circ} \mathrm{C}$, the $n$-type conductivity and oxygen deficiency in $\mathrm{CeO}_{2-x}$ have been alternatively proposed to be due to the cerium interstitials [1-3] or oxygen vacancies [4-6]. However, recent results of $X$-ray and neutron diffraction analysis [7,8], high temperature lattice parameter and macroscopic length change measurements $[9,10]$ and diffusion studies $[11,12]$ have conclusively shown the point defects in $\mathrm{CeO}_{2-x}$ to be the oxygen vacancies. The mobility of the charge carriers in $\mathrm{CeO}_{2-x}$ can be calculated if the carrier concentration and the conductivity can be independently determined. One way of determining the carrier concentration is to measure the nonstojchiometry $x$ in $\mathrm{CeO}_{2-x}$ and calculate the carrier concentration from the known defect structure including the correct ionization state of the defects. Care must be taken to calculate the carrier concentration in the $x$ range where dilute solution model of the defects is valid. This is necessary because the carrier concentration is directly proportional to the point defect concentration, and hence $x$, only in this dilute solution region where defect interactions are negligible. In a recent paper discussing electron mobility in $\mathrm{CeO}_{2-x}[13]$, this was not explicitly done and the ionization state of the defects assumed was not correct as will be discussed later. Also, no attempt was

†This paper is based in part on a dissertation submitted by $\mathbf{I}$. $\mathbf{K}$. Naik in partial fulfillment of the requirements for the Ph.D. degree. The University of Michigan. Ann Arbor (Sept. 1976). made to correlate the experimental results with the known theories of charge transport in low mobility materials. Rectification of these errors from the new experimental data and correlation of the observed results with the small-polaron theory are the main goals of this paper.

\section{THEORY}

The extensive development of small-polaron theory on the basis of the classical Boltzmann transport equation as well as quantum mechanical principles has been reviewed by Appel[14] and Austin and Mott[15]. One of the main conclusions of this theory relevant to this paper is the estimation of the order of magnitude of the smallpolaron drift mobility. The charge transport via smallpolaron motion must carry along the lattice distortion associated with the small-polaron and this imparts additional drag on the motion of the charge carrier. Therefore, the drift mobility of the small-polaron is much smaller compared to that of the carrier in broad band semi-conductors; typically, it is less than $0.1 \mathrm{~cm}^{2} / \mathrm{V} \mathrm{sec} \mathrm{[14].} \mathrm{This} \mathrm{theoretical} \mathrm{expectation} \mathrm{value} \mathrm{of}$ the mobility will serve as the criterion for showing the existence of the small-polarons.

The second conclusion of importance here is that the small-polaron motion in an ionic crystal occurs by two distinct mechanisms. At low temperatures, the smallpolaron moves by Bloch-type band motion while, at elevated temperatures, it moves by thermally-activated hopping mechanisms. This picture applies to the solids having strong coupling between the electrons and lattice vibrations and is expected to be valid for narrow-band, low mobility semiconductors such as transition metal and rare-earth oxides. Holstein and Friedman[16-18] have done the theoretical calculations of small-polaron motion in great detail and shown that the temperature dependences of the small-polaron drift mobility $\left(\mu_{d}\right)$ in the two regimes are different. In the low temperature band motion regime, the drift mobility is mainly determined by the phonon scattering and hence it decreases with increasing temperature in a manner analogous to the 
mobility in broad band semiconductors. In the high temperature hopping regime, the mobility is thermallyactivated and it increases with increasing temperature. As shown by Holstein and Friedman [16, 17], its temperature dependence is characterized by the following equation

$$
\mu_{d}=\frac{\mu_{o}}{T^{3 / 2}} \exp \left(-\frac{E_{m}}{k T}\right)
$$

where $\mu_{o}$ is a constant, $E_{m}$ is the activation energy for mobility, and $T$ is absolute temperature. In this case, the charge of the carrier is localized at a specific site, for example on a cation, and its movement under influence of electric field occurs in discrete jumps from one site to another. This is analagous to the thermally-activated diffusion of atomic species in solids. The hopping mechanism applies if the temperature is greater than (1/2) $\theta_{0}$ where $\theta_{0}$ is the optical Debye temperature given hy

$$
\left.\theta_{0}=\frac{h \omega_{i}}{k} \quad \text { or } \frac{h}{2 \pi}\right)
$$

where $\omega_{i}$ is the longitudinal mode phonon frequency whose typical value for an oxide may be taken as $10^{14}$ per sec. Therefore, hopping-type mobility is expected at temperatures exceeding $500^{\circ} \mathrm{C}$. The transition from the band motion regime to the hopping regime is rather sharp. It occurs in a narrow temperature interval around $(1 / 2) \theta_{i 3}$.

\section{EXPERIMENTAL}

The d.c. electrical conductivity $(\sigma)$ of sintered cerium dioxidet specimens was measured by a four-probe technique over the temperature range $800-1420^{\circ} \mathrm{C}$ and oxygen partial pressure $\left(p_{\mathrm{O}_{2}}\right)$ range $10^{-20}$ to $1 \mathrm{~atm}$. The oxygen pressure was controlled by using $\mathrm{Ar}-\mathrm{O}_{2}$ or $\mathrm{CO}$ $\mathrm{CO}_{2}$ mixtures. At each temperature and $p_{3}$, equilibrium, as indicated by a steady value of conductivity, was established before making the final measurement. Threeprobe conductivity measurements were also carried out to verify that the four-probe measurements yielded the bulk conductivity and not merely the surface or gas conductivity [19]. Electronic transference number of the same specimen was measured by a polarization technique $[19,20]$.

Thermogravimetric analysis by means of a PerkinElmer thermobalance was done to determine the nonstoichiometry at different temperatures and $p_{\mathrm{O}_{2}}$. The weight changes were measured relative to the weight of the specimen at $1000^{\circ} \mathrm{C}$ in air. Pure $\mathrm{CeO}_{2}$ is essentially stoichiometric under this condition as far as weight change measurements are concerned.

Further details of the experimental procedure can be found in Ref. [19].

\section{RESULTS AND DISCUSSION}

The electronic transference number measurements showed that $\mathrm{CeO}_{2-x}$ is predominantly an electronic

THigh purity $\mathrm{CeO}_{2}$ from Kerr-McGee Chem, Corp; $\mathrm{Ce}$ content $99.99 \%$ of total rare earth; main non-rare earth impurities: $\mathrm{Mg}, \mathrm{Ca}$ and $\mathrm{Si}$, all in trace amounts. conductor. It has been demonstrated by Brugner and Blumenthal[21] that the conductivity of single crystal $\mathrm{CeO}_{2-x}$ specimen and the normalized conductivity $\left(\sigma_{\text {norm }}\right)$ of sintered polycrystalline $\mathrm{CeO}_{2-x}$ specimen are identical. Here, $\sigma_{\text {norm }}=\sigma_{\text {measured }}\left(\rho_{\text {theoretical }} / \rho_{\text {measured }}\right), \rho$ being the densities; it takes into account the porosity effect. This means that the extra electron scattering from the grain boundaries and pores in the sintered specimens is negligible. This is expected for $\mathrm{CeO}_{2-x}$ since, as shown later, it is a low mobility oxide semiconductor in which the mean free path of the charge carriers is small. Therefore, the conductivity of the sintered specimens, after normalizing for porosity, can be taken to represent the true conductivity of $\mathrm{CeO}_{2-x}$ crystal. As mentioned in the introduction, the predominant defects in $\mathrm{CeO}_{2-x}$ are oxygen vacancies. To get the ionization state of these oxygen vacancies, the conductivity variation with $p_{\mathrm{O}_{2}}$ shown in Fig. 1 was analyzed in the light of the quasichemical defect theory based on a dilute solution model of the defects in oxides[22]. The slopes of $\log \sigma$ vs $\log \mathrm{p}_{2}$ curves indicate the ionization state of the oxygen vacancies. At high oxygen pressures, i.e. for small $x$ values, the least square straight line slopes are close to $-1 / 6$ which means the oxygen vacancies are doublyionized. This is valid up to $x$ value of about 0.007 . As $x$ increases beyond this, the proportion of the singly-ionized vacancies increases as indicated by the slope changing to $-1 / 5$ and $-1 / 4$ with decreasing $p_{\mathrm{O}_{2}}$. In the $x$ range $0.01-0.04$, the oxygen vacancies are predominantly singly-ionized. This defect model will be used to calculate the drift mobility from the conductivity and nonstoichiometry data. At $x$ values greater than 0.04 , the dependence of the conductivity on $\log p_{\mathrm{O}_{2}}$, and hence on $x$, is complex. This is interpreted as a situation where the defect theory based on the dilute solution model is not

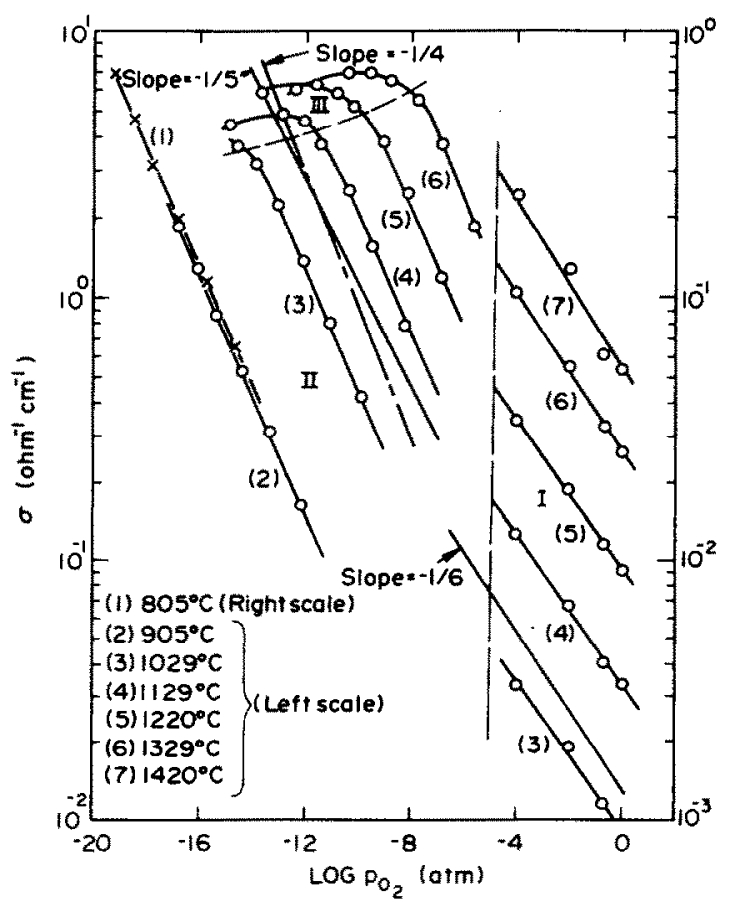

Fig. 1. Oxygen partial pressure dependence of electrical conductivity of $\mathrm{CeO}_{2-x}$ at constant temperature. 
applicable due to the interaction or partial ordering of the vacancies.

The conductivity and nonstoichiometry data can now be combined to calculate $\mu_{d}$ on the basis of the above defect model in the $x$ range where a single state of ionization predominates. In the conductivity equation,

$$
\sigma=n e \mu_{d}
$$

where $e=$ electronic charge, the charge carrier concentration $(n)$ is directly proportional to $x$ if the dilute solution model is valid. Then, for singly-ionized oxygen vacancies, $\mu_{d}$ is given by,

$$
\mu_{d}=2.482 \times 10^{-4}\left(\frac{\sigma}{x}\right)
$$

where $\mu_{d}$ is the drift mobility in $\mathrm{cm}^{2} / \mathrm{V}$ sec and $\sigma$ is the conductivity in $\mathrm{ohm}^{-1} \mathrm{~cm}^{-1}$. As shown in Fig. 2, $\sigma$ varies linearly with $x$ up to $x=0.03$ indicating the validity of the dilute solution model. A single straight line can be drawn through the experimental points up to $x=0.03$ regardless of temperature. This means that $\mu_{d}$ does not change much with temperature up to $x=0.03$. Calculated $\mu_{d}$ values for $x$ close to $\mathbf{0 . 0 2 5}$, for which singly-ionized oxygen vacancies predominate, are plotted on a logarithmic scale against $1 / T$ in Fig. 3.

The small magnitude of $\mu_{d}$ indicates that the charge carriers in $\mathrm{CeO}_{2-\mathrm{X}}$ are small-polarons. These smallpolarons would be formed by localization of electrons at cerium cations. This is equivalent to reduction of some $\mathrm{Ce}^{4+}$ ions to $\mathrm{Ce}^{3+}$ state. The relatively high temperatures $\left(T>1 / 2 \theta_{0}\right)$ involved here would lead one to expect

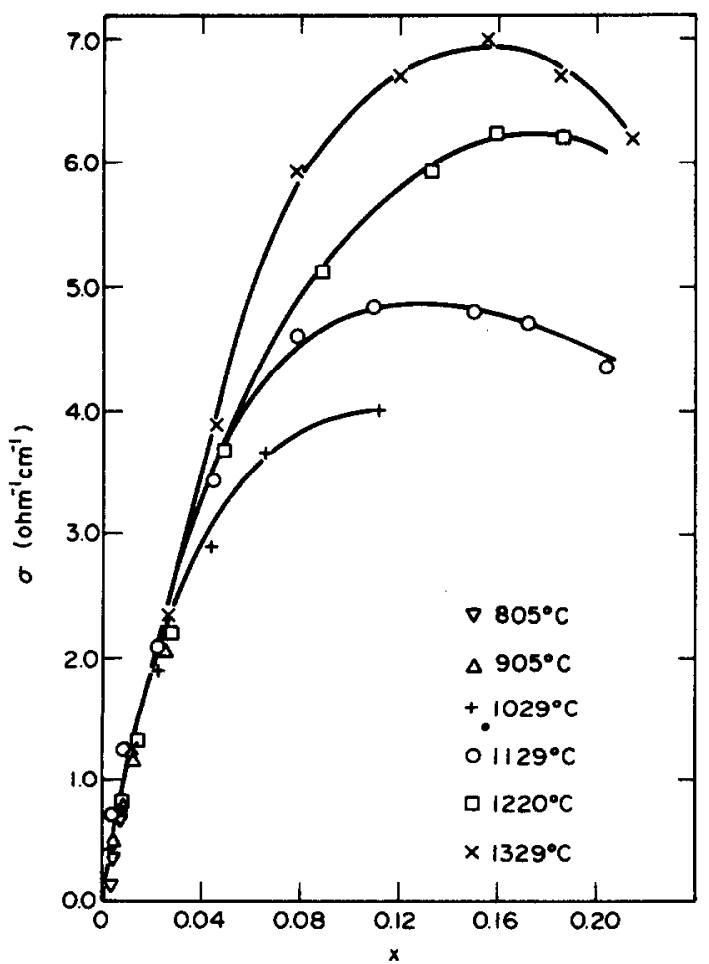

Fig. 2. Electrical conductivity vs nonstoichiometry in $\mathrm{CeO}_{2-x}$.

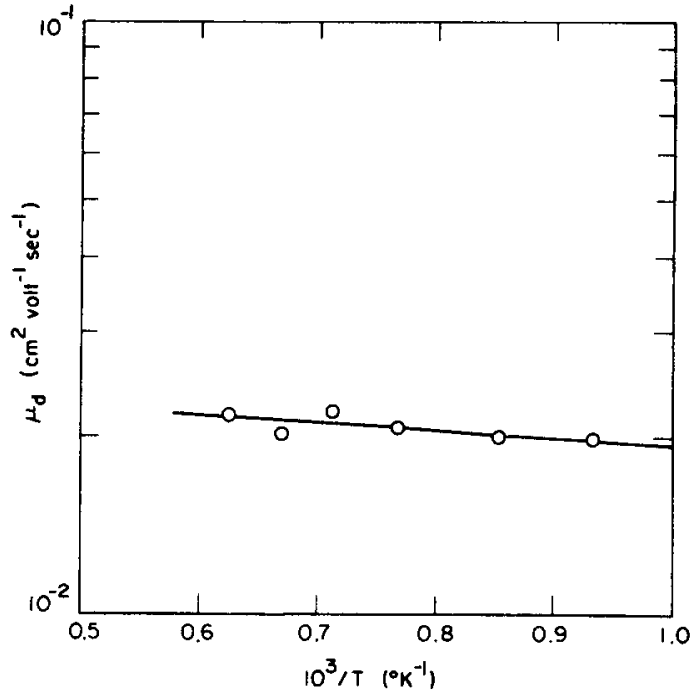

Fig. 3. Drift mobility vs $1 / T$ for $x=0.025$.

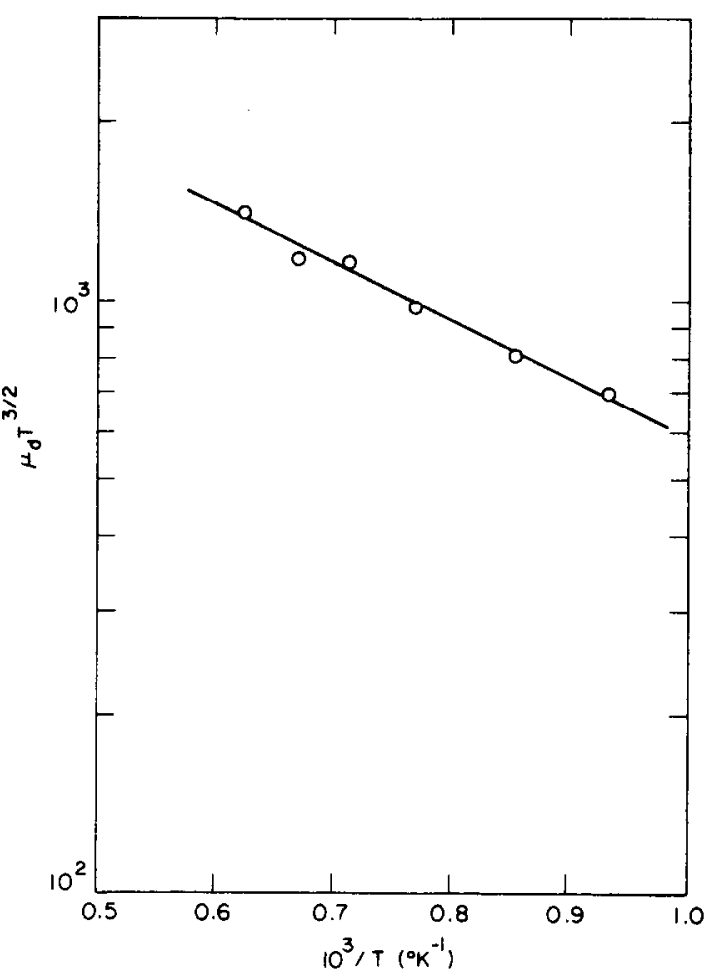

Fig. 4. $\mu_{d} T^{3 / 2}$ vs $1 / T$ for $x=0.025$.

hopping-type small-polaron motion on the basis of the theory discussed in Section 2. The temperature dependence of the mobility can indeed be explained on the basis of eqn (1). In this equation, the exponential factor and $T^{3 / 2}$ factor both increase with increasing temperature. Therefore, in the temperature range $800-1400^{\circ} \mathrm{C}, \mu_{d}$ increases very slowly with increasing temperature if $E_{m}$ is between 0.15 and $0.20 \mathrm{eV}$. On the other hand, smallpolaron transport by band motion requires that the mobility decrease with increasing temperature. Figure 3 shows that the experimentally determined $\mu_{d}$ increases slightly with temperature for $x$ close to 0.025 . Next, $\mu_{d} T^{3 / 2}$ is plotted vs $1 / T$ in Fig. 4 . Least square analysis 
showed this to be linear and, hence in accordance with eqn (1). Therefore, the small-polaron motion must occur by a hopping mechanism. The hopping would involve electrons jumping from $\mathrm{Ce}^{3+}$ ion to a neighboring $\mathrm{Ce}^{4+}$ ion. The activation energy of mobility $\left(E_{m}\right)$ is $0.19 \mathrm{eV}$ and it is independent of $x$ up to $x=0.03$.

Now, the mobility eqn (1) can be applied in the $x$ range where $\sigma$ does not vary linearly with $x$, i.e. for $x>0.03$, with the assumption that $E_{n}$ is a function of $x$. This means that the dependence of the mobility on $x$ is nonlinear. It is also reasonable to assume that the carrier concentration $(n)$ is determined by $x$ alone. In this case,

$$
\begin{aligned}
\sigma(x, T) & =e n(x) \mu_{d}(x, T) \\
& =e n(x)\left[\frac{\mu_{0}}{T^{3 / 2}} \exp \left(-\frac{E_{m}(x)}{k T}\right)\right]
\end{aligned}
$$

Therefore, for a tixed $x$,

$$
\sigma(T)=\frac{\sigma_{0}}{T^{3 / 2}} \exp \left(-\frac{E_{m}(x)}{k T}\right)
$$

Equation (6) shows that, if $\log \left(\sigma T^{3 / 2}\right)$ is plotted vs $1 / T$ for a fixed value of $x$, a straight line will be obtained and the slope of this line can be used to calculate $E_{m}(x)$. Figure 5 presents such plots of the experimental data. Good linear plots of $\log \left(\sigma T^{3 / 2}\right)$ vs $1 / T$ were also obtained for $0.12<x<0.20$; however, the points for $x>0.12$ have been omitted from the figure for the sake of clarity. $E_{m}(x)$, calculated from the least square straight lines corresponding to different $x$ values, is plotted as a function of $x$ in Fig. 6. It is seen that $E_{m}(x)$ increases and therefore $\mu_{d}$ decreases with increasing $x$. This is a direct result of the high defect concentrations

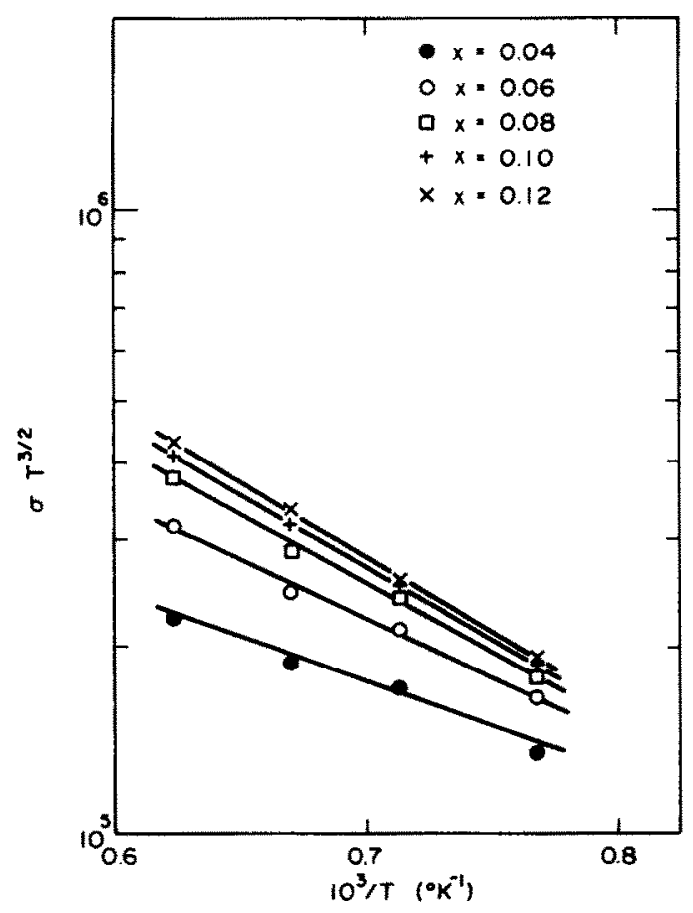

Fig. 5. $\log \left(\sigma T^{3 / 2}\right)$ vs $1 / T$ at constant $x$.

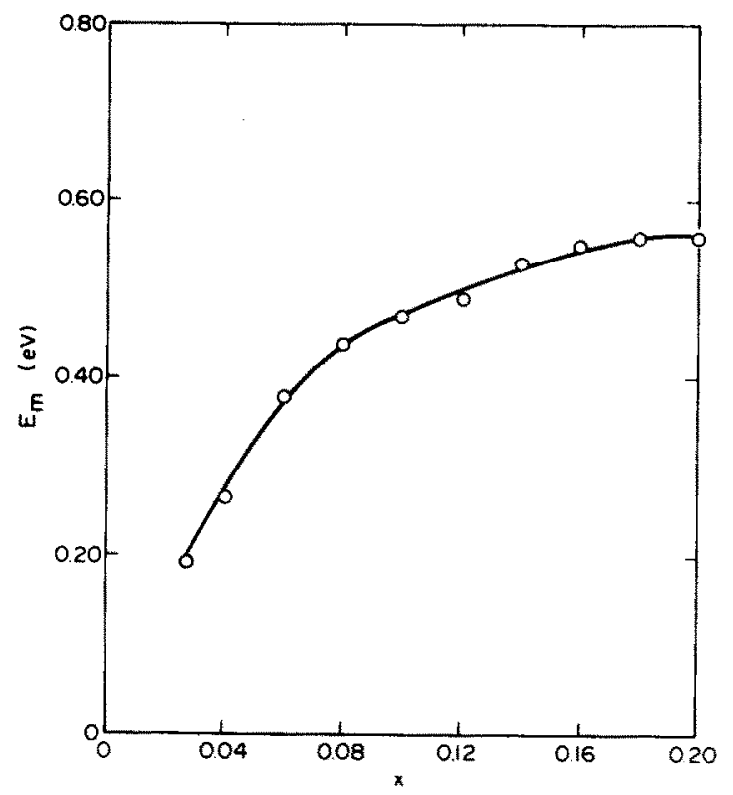

Fig. 6. Activation energy of mobility vs $x$.

occurring at the large departures from stoichiometry. When the defect concentration is high, the electrons are not likely to move randomly independent of the surrounding point defects as assumed in the dilute solution hopping model. Any localized electron will be surrounded by a significant number of similarly charged defects at a close distance. Thus, this localized electron will experience repulsive forces from the surrounding defects of similar charge. These repulsive forces would limit the number of directions in which the electron can jump reducing its mobility as a result. Hence, the observed increase in $E_{m}$ (or decrease in $\mu_{d}$ ) with increasing $x$ is expected for the hopping motion at large defect concentrations.

\section{CONCLUSION}

In nonstoichiometric $\mathrm{CeO}_{2-x}$, the electrons are localized and the high temperature electrical conduction occurs by a hopping mechanism in which electrons jump from a $\mathrm{Ce}^{3+}$ ion to a neighboring $\mathrm{Ce}^{4 t}$ ion. The smallpolaron model developed by Holstein and Friedman satisfactorily explains the observed temperature dependence of the high temperature drift mobility. The activation energy of mobility $\left(E_{m}\right)$ has been calculated as a function of nonstoichiometry $(x)$. It has been found that $E_{m}$ increases with increasing $x$ which is in accord with the hopping model.

Addendum: While the manuscript of this paper was being prepared, a related paper entitled "Small Polaron Electron Transport in Reduced $\mathrm{CeO}_{2}$ Single Crystals" was submitted to this journal by Tuller and Nowick [23]. It reports the results of Seebeck coefficient and electrical conductivity measurements on reduced $\mathrm{CeO}_{2-x}$ obtained by sealing off the specimen chamber after reduction to a specific $x$ value. The Seebeck coefficient was found to be independent of temperature for a fixed $x$, viz. $x=0.008$, 0.040 and 0.200 . This indicated a hopping mechanism of charge transport in light of the small-polaron theory. The 
present paper arrives at the hopping mechanism of smallpolaron transport in $\mathrm{CeO}_{2-x}$ on the basis of the absolute value and temperature dependence of the charge carrier mobility which has been obtained by combining the equilibrium conductivity and nonstoichiometry data. The mobility values in the two papers are comparable, viz. at $T=1000^{\circ} \mathrm{C}$ and $x \simeq 0.025, \mu_{d}=0.02 \mathrm{~cm}^{2} / \mathrm{V}$ sec in the present paper and $0.007 \mathrm{~cm}^{2} / \mathrm{V}$ sec in the Tuller and Nowick paper. Their measurements on the sealed-off specimens may make the data dependent on the history of the measurements to some extent since oxidation or reduction of $\mathrm{CeO}_{2-x}$ could occur during the measurements depending on the $\mathrm{P}_{2}$ value of the atmosphere surrounding the specimen. This factor is probably responsible for the difference in the values of the activation energy of mobility $\left(E_{m}\right)$ for low $x$ where the greatest possibility of change in $x$ exists, viz. for $0.01<x<0.06$, $E_{m}$ increasing from $0.20 \mathrm{eV}$ to $0.40 \mathrm{eV}$ in the present paper and $E_{m}=0.40 \mathrm{eV}$ in the Tuller and Nowick paper. At larger $x$ values, $E_{m}$ values in the two papers are in good agreement.

Acknowledgement-Financial support from the General Motors Corporation is gratefully acknowledged.

\section{REFERENCES}

1. Greener E. H., Wimmer J. M. and Hirthe W. M., In Rare Earth Research II (Edited by K. S. Vorres), p. 539. Gordon \& Breach, New York (1964).

2. Blumenthal R. H., Lee P. W. and Panlener R. J., J. Electrochem. Soc. 118, 123 (1971).

3. Kofstad P. and Hed A. Z., J. Am. Ceram. Soc. 50, 691 (1967).
4. Kevane C. J., Phys. Rev. 133, Al431 (1944).

5. Blumenthal R. N. and Laubach J. E., In Anisotropy in Single Crystal Refractory Compounds (Edited by F. W. Vahldiek and S. A. Merson), Vol. 2, p. 137. Plenum Press, New York (1968).

6. Panlener R. J., Blumenthal R. N. and Garnier J. E., J. Phys. Chem. Solids 36, 1213 (1975).

7. Faber J., Seitz M. A. and Mueller M. H., J. Phys. Chem. Solids 37, 903 (1976).

8. Faber J., Seitz M. A. and Mueller M. H., J. Phys. Chem. Solids 37, 909 (1976).

9. Ban Y. and Nowick A. S., Proc. 5th Materials Res. Symp. N.B.S. special publication No. 364, p. 353 (1972).

10. Sims J. R. Jr., Ph.D. Dissertation, Marquette University, Milwaukee, Wisconsin, U.S.A. (1973).

11. Steele B. C. H. and Floyd J. M., Proc. Br. Ceram. Soc. 19, 55 (1971).

12. Floyd J. M., Indian J. Tech. 11, 589 (1973).

13. Blumethal R. N. and Sharma R. K., J. Solid State Chem. 13, 360 (1975).

14. Appel J., In Solid State Physics (Edited by F. Seitz and D. Turnbull), Vol. 21, p. 193. Acadentic Press, New York (1968).

15. Austen I. G. and Mott N. F., Adv. Phys. 18, 41 (1969).

16. Holstein T., Ann. Phys. 8, 343 (1959).

17. Friedman L. and Holstein T., Ann. Phys. 21, 494 (1963).

18. Friedman L., Phys. Rev. 133A, 1668 (1964); 135A, 233 (1964).

19. Naik I. K., Ph.D. Dissertation, The University of Michigan, Ann Arbor, Michigan, U.S.A. (1976).

20. Vest R. W. and Tallan N. M., J. Appl. Phys. 36, 543 (1965).

21. Brugner F. S. and Blumenthal R. N., J. Am. Ceram. Soc. 54, 57 (1971).

22. Kofstad P., Nonstoichiometry, Diffusion and Electrical Conductivity in Binary Metal Oxides. Wiley-Interscience, New York (1972).

23. Tuller H. L. and Nowick A. S., J. Phys. Chem. Solids. 38, 859 (1977). 